\title{
Numerical Simulation Analysis and Test of Standing Wave Border Speed about High-speed Radial Tire
}

\author{
Wang Qiang $^{1, a}$, Qi Xiaojie ${ }^{2, b}$ \\ ${ }^{1}$ HeiLongjiang Institute of Technology, HarBin, 150050, China \\ ${ }^{2}$ HeiLongjiang Institute of Technology, HarBin, 150050, China \\ aemail: 630702666@163.com, ${ }^{\mathrm{b}} \mathrm{qxj}$ 725@sina.com
}

\begin{abstract}
Keywords: Radial Tire; Standing Wave; Border Speed; Finite Element; Numerical Simulation component
\end{abstract}

\begin{abstract}
The standing wave phenomenon of radial tire occurs when the vehicle speed reaches a certain critical value, this phenomenon can easily result in traffic accidents. In this paper, it completed numerical simulation analysis of standing wave border speed about 195/60R15 radial tire by use of ABAQUS finite element analysis software, and determined characterization methods of standing wave border speed about radial tire, and then realized the experimental verification by use of a test system. Based on these, it concludes that those measures to advance the tire pressure and reduce the tire load can increase the border speed value of standing wave effectively, which not only presents border speed indicators for the safe driving of vehicle, but also provides a scientific basis for the design and manufacture of tire that is able to resist high speed and traffic management.
\end{abstract}

\section{Introduction}

With the advancement of vehicle speed, wave frequency and wave distortion of the radial tire crown surface increases and the tire standing wave phenomenon occurs when the vehicle speed reaches a certain critical point. After the standing wave happens, the thermal deformation, circumferential deformation and lateral distortion deformation of the tire increase dramatically, and the temperature of carcass rises rapidly, and the material properties decline quickly, which might cause severe puncture accidents. Early literatures that researched standing wave phenomena were more concentrated on the analytical methods, which was difficult to fully take the impact on the generation of standing wave into account and these influence factors mainly include the various parts of the tire structure and material properties and so on. In recent years, non-linear finite element technology has been widely used in tire mechanics analysis[1]. For the purpose of further improvement of traffic safety and promotion of the tire manufacturing industry, by combing ABAQUS finite element analysis software simulation with standing wave test, this paper conducts the numerical simulation analysis and test of high-speed tire standing wave. Based on these, the characterization method of the tire standing wave critical speed is determined and the critical speed indicators for vehicle safe driving are provided.

\section{The standing wave critical speed of high-speed tire}

Many scholars have conducted a theoretical study on the critical speed of tire standing wave[2-3].

(1) As early as 1954, Turner predicted approximately the critical velocity and it could be expressed using the following formula:

$$
C=\sqrt{\frac{T}{\rho}}
$$

Where $\mathrm{C}$ is the propagation velocity of wave, and it is the critical speed when the standing wave occurs; $T$ is circumferential tension of tire and its size depends on the combination of inflation pressure and speed; ${ }^{\rho}$ is density of tread material.

(2) The radial tire was simulated as radial and vertical spring support in the model formula of 
elastic base ring beam used by Zhuang Jide, that is to say, this model only took into account the radial damping of tire except the bending stiffness of tread and longitudinal stiffness of tire and advanced a more sophisticated argument and modification. The formula was expressed as:

$$
V_{c}=\frac{\frac{E_{J}}{r^{2}} a^{2}\left(a^{2}+r^{2}-1\right)^{2}+T a^{4} r^{2}+\left(r^{2} C_{r}-T\right) a^{2}+C_{t}}{\rho a^{2}\left(a^{2} r^{2}+6\right)}
$$

Where $E_{J}$ is the bending stiffness of tread; $r$ is the outside radius of tire; $\rho$ is the mass per unit length of ring beam; $T$ is the tension of belt; $C_{r}$ is the radial stiffness per unit length of tire; $C_{t}$ is the longitudinal stiffness per unit length of tire; $a$ is the wave number, $a=2 \pi / \lambda$ ( $\lambda$ is the wave length).

(3) Deformation and recovery of tire circumference occur repeatedly when the rotation. When vehicle travels at a high speed, the rotation speed of tire becomes faster. As deformation (wrinkles) occurred in the ground has not been recovered during the period including the time of a circle rotation and the time of contact with the ground again, the deformation wrinkles are left in the circle and tires produce standing waves. Therefore, it can be seen that standing waves occur when tire deformation is repeated, and the condition of deformation is that the tire has turned a circle in the deformation recovery time, and more exactly to say, standing waves occur when deformation superimposed with the next. Thus, when the recovery time $\mathrm{T}$ of tire is greater than or equal to the time $\mathrm{t}$ used by a circle rotation, standing waves occur. Here, fixed at an angle $\alpha$, the tire deformation fully restores at the speed of $\mathrm{V} \mathrm{km} / \mathrm{h}[4-5]$.

As $v=2 V$ and $T=t$, then formula (7) is obtained, as shown:

$$
t=2 \pi r / v
$$

So formula (8) is expressed as shown:

$$
V=\pi r / T
$$

Where $\mathrm{V}$ is the critical speed; $\mathrm{v}$ is the speed of wheel rotation; $\mathrm{r}$ is the rolling radius; $\mathrm{T}$ is the recovery time.

\section{Tire standing wave critical speed by use of FEM}

1) Construction of finite element model [6]

Tread, anti-rub line and mark line were ignored when constructing the geometric model, and the contact between the tire and rim was simplified as the fixed constraint of the bead corresponding region and rim was no longer defined. And then tire and drum were assembled and the location was restricted. Finally, material behavior was super-elastic and strain potential options were Yeoh.

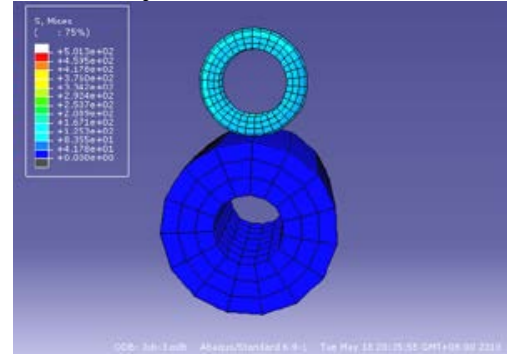

Fig.1 Deformation stress cloud figure when $150 \mathrm{~km} / \mathrm{h}$

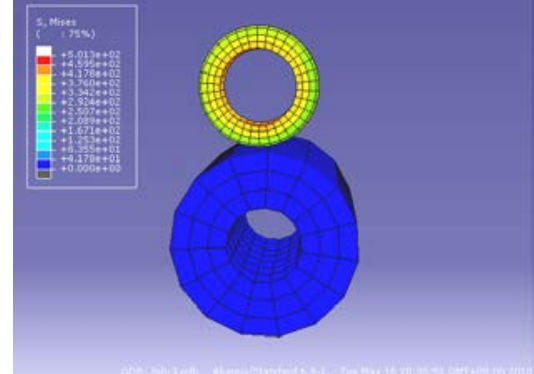

Fig.3 Deformation stress cloud figure when $170 \mathrm{~km} / \mathrm{h}$

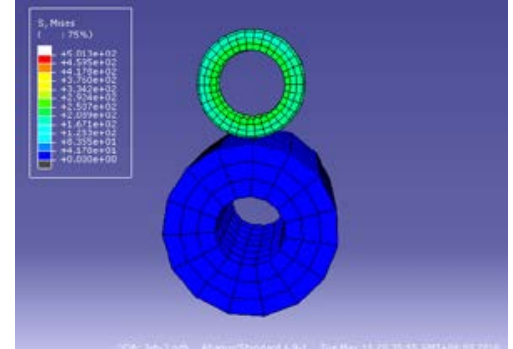

Fig.2 Deformation stress cloud figure when $160 \mathrm{~km} / \mathrm{h}$

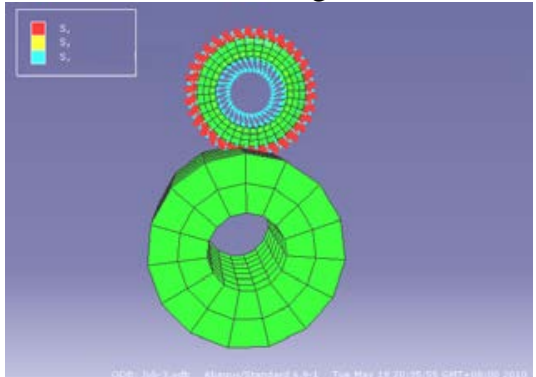

Fig.4 Deformation stress cloud figure when $180 \mathrm{~km} / \mathrm{h}$ 


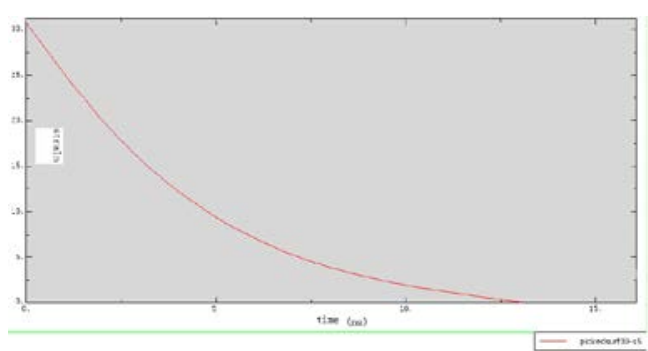

Fig.5 Deformation recovery schematic diagram of tread nodes

2) Conclusion of numerical simulation

The stress cloud figure of rotational deformation was respectively shown in Fig.1,2,3 and 4 when the speed were chosen as $150 \mathrm{~km} / \mathrm{h}, 160 \mathrm{~km} / \mathrm{h}, 170 \mathrm{~km} / \mathrm{h}, 180 \mathrm{~km} / \mathrm{h}$ respectively. The result of strain and time was shown in Fig.5 and deformation recovery time of simulation was $0.01311 \mathrm{~s}$.

3) Calculation of the critical speed

By use of the formula (4), the result is as shown in: $V=\frac{\pi r}{T}=\frac{3.14 \times(0.307-0.0103)}{0.01311}=255.83 \mathrm{~km} / \mathrm{h}$

\section{The critical speed test and analysis of the tire standing wave}

(1) Test equipments

Test equipments mainly included a toyota corolla car (195/60 R15 radial tires), a chassis dynamometer, a high-speed camera, a desktop computer controlled by high-speed camera, a silver pen and other auxiliary devices.

(2) Experimental procedures

1) Marked the reference point of tire; 2) The car was fixed on the chassis dynamometer;3) High-speed camera was installed, and the camera lens was level with the tire center, and the camera direction was plumb with the tire plane; 4) The car on chassis dynamometer travelled at a constant speed and the speed range was chosen from $150 \mathrm{~km} / \mathrm{h}$ to $180 \mathrm{~km} / \mathrm{h}$, and the speed interval tested was $10 \mathrm{~km} / \mathrm{h}$; 5) The functioning state of tire on the chassis dynamometer was obtained by camera and the radial deformation of tire was recorded at the different speed value; 6) At the same time, the deformation angle when tire being in the complete response state of elastic deformation and time were observed, and the deformation angle mentioned above was formed with the vertical direction by the complete response point connected with axis;7) Experiment data was collected using the high-speed camera; 8) the recorded images were imported into AutoCAD and calculated.

(3) Results analysis

The curves of the tire deformation were shown in Fig.6, 7, 81 and 9 when the vehicle speed was $150 \mathrm{~km} / \mathrm{h}, 160 \mathrm{~km} / \mathrm{h}, 170 \mathrm{~km} / \mathrm{h}$ and $180 \mathrm{~km} / \mathrm{h}$ respectively.

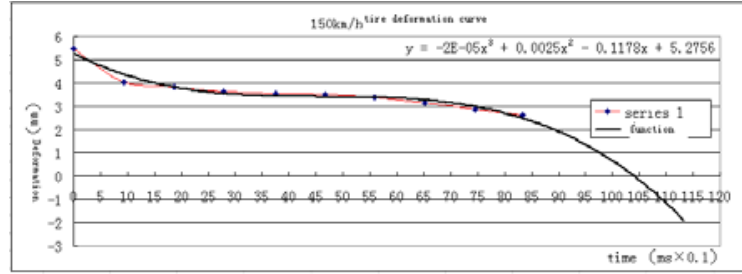

Fig.6 the tire deformation curve at the speed of $150 \mathrm{~km} / \mathrm{h}$

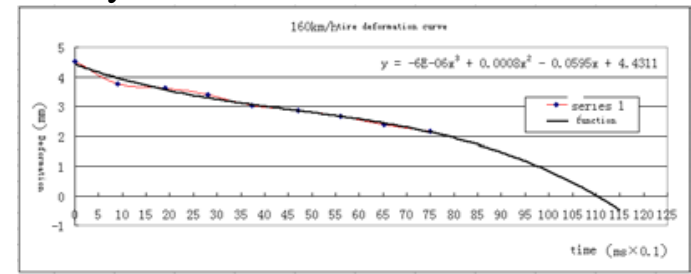

Fig,7 the tire deformation curve at the speed of $160 \mathrm{~km} / \mathrm{h}$

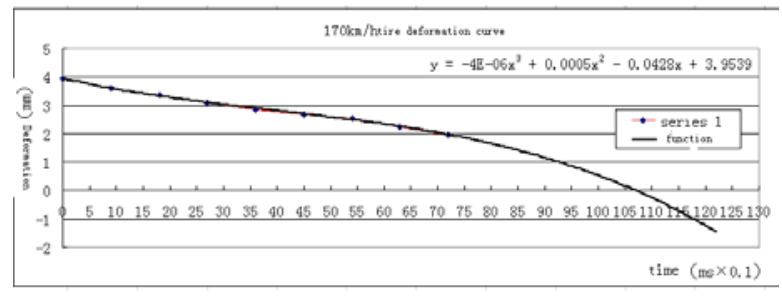

Fig.8 the tire deformation curve at the speed of $170 \mathrm{~km} / \mathrm{h}$

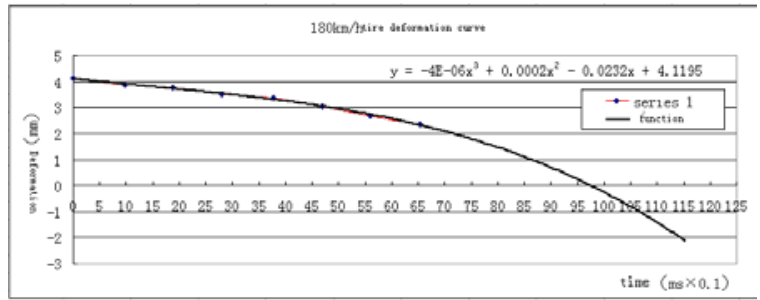

Fig.9 the tire deformation curve at the speed of $180 \mathrm{~km} / \mathrm{h}$

(4) Calculation of standing wave critical speed

It was showed from the figures obtained above that the recovery time of fitting function was 
$\mathrm{T} 1=0.0104 \mathrm{~s}, \mathrm{~T} 2=0.0111 \mathrm{~s}, \mathrm{~T} 3=0.0108 \mathrm{~s}$ and $\mathrm{T} 4=0.0098 \mathrm{~s}$ when the speed was $150 \mathrm{~km} / \mathrm{h}, 160 \mathrm{~km} / \mathrm{h}$, $170 \mathrm{~km} / \mathrm{h}$ and $180 \mathrm{~km} / \mathrm{h}$ respectively. And then according to formula (10):

$$
t=\frac{t_{z}}{\alpha_{z}-\alpha_{1}}
$$

$\mathrm{t}_{1}=0.002049, \mathrm{t}_{2}=0.002115, \mathrm{t}_{3}=0.001939$ and $\mathrm{t}_{4}=0.001861$ can be concluded. And based on this, the result is expressed.

$$
\begin{aligned}
& T_{\text {平均 }}=\frac{\left(T_{1}+t_{1}\right)+T_{2}+t_{2}+T_{3}++t_{3}+T_{4}+t_{4}}{4} \\
& =0.012449+0.013215+0.012739+0.011661 \\
& =0.012516 \mathrm{~s}
\end{aligned}
$$

Where $\mathrm{t}$ is the previous recovery time of the No.1 point marked; $t_{z}$ is the total time; $\alpha_{1}$ is angle of the No.1 point marked; $\alpha_{z}$ is angle of the last point marked; $T$ is recovery time from the No.1 to the last.

$$
\begin{gathered}
T_{\text {average }}=T=0.012516 \mathrm{~s} \\
r=R-H / 3=0.307-0.0097=0.2973 \mathrm{~m}
\end{gathered}
$$

According to the formula (8), the result was

$$
V=\frac{\pi r}{T}=\frac{3.14 \times 0.2973}{0.012516}=268.5 \mathrm{~km} / \mathrm{h}
$$

which shows that the test value is slightly larger than the simulation value.

\section{Summary}

Based on the combined research results from domestic and foreign scholars, this paper made the use of ABAQUS finite element software to complete the numerical simulation of the standing wave critical speed and established the test system of critical speed. The results showed that the simulation value was consistent with the test value and the calculated value. The following conclusions are presented that firstly the critical speed of 195/60R15 radial tires is $268.5 \mathrm{~km} / \mathrm{h}$ under the pressure of $1.2 \mathrm{MPa}$, and the load of $280 \mathrm{KG}$ and the outside temperature of $20^{\circ} \mathrm{C}$ condition; Secondly, when the load is fixed, the radial deformation can be reduced and flexible recovery time can be also shortened by advancing the pressure of tire, thereby increasing the critical speed; Thirdly, under a certain inflation pressure, the reduction of the load can help improve the critical velocity which have not linear correlations with occurrence of standing wave.

\section{References}

[1] Zhuang Jide. Calculate vehicle ground mechanics [M]. Beijing: Mechanical Industry Press, 2005.

[2] Zhuang Jide Modern vehicle tire technology [M]. Beijing: Beijing University of Technology Press, 2001.

[3] Padovan J. On standing waves in tires [J]. Tire Science and Technology, 1997, 5(2).

[4] J.R.Choa, K.W.Kimb, H.S.Jeongb. Numerical investigation of tire standing wave using 3-D patterned tire model[J]. Science Direct, 2007(6).

[5] Aninda Chatterjee, Joseph P.Cusumano and John D.Zolock. On contact-induced standing waves in rotating tires:experiment and theory[J]. Journal of Sound and Vibration, 1999(5).

[6] Hong Zongyue, Wu Guizhong. Finite element analysis of radial tire. lecture 6 finite element analysis model of radial tire [J]. Tire Industry, 2006 (3) :187-191. 